\title{
A Note on Non-commutativity
}

\author{
Valeri V. Dvoeglazov \\ Universidad de Zacatecas \\ Apartado Postal 636, Suc. 3 \\ Zacatecas 98061 Zacatecas, México \\ E-mail: valeri@fisica.uaz.edu.mx
}

\begin{abstract}
ABSTRACT. Ambiguities have recently been found in the definition of the partial derivative (in the case of presence of both explicit and implicit dependencies of the function subjected to differentiation). We investigate the possible influence of this subject on quantum mechanics and the classical/quantum field theory. Surprisingly, some commutators of operators of space-time 4-coordinates and those of 4-momenta are not equal to zero.

RESUMÉ. Des ambiguïtés ont été récemment trouvées dans la définition de la dérivée partielle (dans le cas de présence de dépendances à la fois explicites et implicites de la fonction soumise à la différenciation). Nous étudions l'influence possible de ce sujet sur la mécanique quantique et la théorie classique / quantique des champs. Fait surprenant, certains commutateurs d'opérateurs de coordonnées spatio-temporelles et ceux de 4-moments ne sont pas égaux à zéro.

KEYWORDS: Non-commutativity, quantum mechanics, whole-partial derivatives
\end{abstract}

PACS: 04.62.+v 02.40.Gh 02.30.-f

The assumption that the operators of coordinates do not commute $\left[\hat{x}_{\mu}, \hat{x}_{\nu}\right] \neq$ 0 has been made by H. Snyder [1]. The Lorentz symmetry thus may be broken. This idea $[2,3]$ received attention in the context of "brane theories". Moreover, the famous Feynman-Dyson proof of Maxwell equations [4] contains intrinsically the non-commutativity of velocities $\left[\dot{x}_{i}(t), \dot{x}_{j}(t)\right] \neq 0$ that 
also may be considered as a contradiction with the well-accepted theories (while there is no any contradiction therein).

On the other hand, it was recently discovered that the concept of partial derivative is not well defined in the case of both explicit and implicit dependence of the corresponding function, which the derivatives act upon [5]. The well-known example of such a situation is the field of an accelerated charge [6]. ${ }^{1}$ Škovrlj and Ivezić [7] call this partial derivative as 'complete partial derivative'; Chubykalo and Vlayev, as 'total derivative with respect to a given variable'. The terminology suggested by Brownstein [5] is 'the whole-partial derivative'.

Let us study the case when we deal with explicit and implicit dependencies $f(\mathbf{p}, E(\mathbf{p}))$. It is well known that the energy in the relativism is connected with the 3-momentum as $E= \pm \sqrt{\mathbf{p}^{2}+m^{2}}$; the unit system $c=\hbar=1$ is used. In other words, we must choose the 3-dimensional hyperboloid from the entire Minkowski space and the energy is not an independent quantity anymore. Let us calculate the commutator of the whole derivative $\hat{\partial} / \hat{\partial} E$ and $\hat{\partial} / \hat{\partial} p_{i}$. In order to make distinction between differentiating the explicit function and that which contains both explicit and implicit dependencies, the 'whole partial derivative' may be denoted as $\hat{\partial}$. In the general case one has

$$
\frac{\hat{\partial} f(\mathbf{p}, E(\mathbf{p}))}{\hat{\partial} p_{i}} \equiv \frac{\partial f(\mathbf{p}, E(\mathbf{p}))}{\partial p_{i}}+\frac{\partial f(\mathbf{p}, E(\mathbf{p}))}{\partial E} \frac{\partial E}{\partial p_{i}} .
$$

Applying this rule, we surprisingly find

$$
\begin{array}{r}
{\left[\frac{\hat{\partial}}{\hat{\partial} p_{i}}, \frac{\hat{\partial}}{\hat{\partial} E}\right] f(\mathbf{p}, E(\mathbf{p}))=\frac{\hat{\partial}}{\hat{\partial} p_{i}} \frac{\partial f}{\partial E}-\frac{\partial}{\partial E}\left(\frac{\partial f}{\partial p_{i}}+\frac{\partial f}{\partial E} \frac{\partial E}{\partial p_{i}}\right)=} \\
=\frac{\partial^{2} f}{\partial E \partial p_{i}}+\frac{\partial^{2} f}{\partial E^{2}} \frac{\partial E}{\partial p_{i}}-\frac{\partial^{2} f}{\partial p_{i} \partial E}-\frac{\partial^{2} f}{\partial E^{2}} \frac{\partial E}{\partial p_{i}}-\frac{\partial f}{\partial E} \frac{\partial}{\partial E}\left(\frac{\partial E}{\partial p_{i}}\right) .
\end{array}
$$

So, if $E= \pm \sqrt{m^{2}+\mathbf{p}^{2}}$ and one uses the generally-accepted representation form of $\partial E / \partial p_{i}=p_{i} / E$, one has that the expression (2) appears to be equal

\footnotetext{
${ }^{1}$ Firstly, Landau and Lifshitz wrote that the functions depended on $t^{\prime}$, and only through $t^{\prime}+R\left(t^{\prime}\right) / c=t$ they depended implicitly on $x, y, z, t$. However, later (in calculating the formula (63.7)) they used the explicit dependence of $R$ on the space coordinates of the observation point too. Jackson [8] agrees with [6] that one should find "a contribution to the spatial partial derivative for fixed time $t$ from explicit spatial coordinate dependence (of the observation point)."
} 
to $\left(p_{i} / E^{2}\right) \frac{\partial f(\mathbf{p}, E(\mathbf{p}))}{\partial E}$. Within the choice of the normalization the coefficient may be related to the longitudinal electric field in the helicity basis (the electric/magnetic fields can be derived from the 4-potentials which have been presented in [9]). On the other hand, the commutator

$$
\left[\frac{\hat{\partial}}{\hat{\partial} p_{i}}, \frac{\hat{\partial}}{\hat{\partial} p_{j}}\right] f(\mathbf{p}, E(\mathbf{p}))=\frac{1}{|E|^{3}} \frac{\partial f(\mathbf{p}, E(\mathbf{p}))}{\partial E}\left[p_{i}, p_{j}\right] .
$$

This should also not be zero according to Feynman and Dyson [4]. They postulated that the velocity (or, of course, the 3-momentum) commutator is equal to $\left[p_{i}, p_{j}\right] \sim i \hbar \epsilon_{i j k} B^{k}$, i.e., to the magnetic field. In fact, if we put in the corespondence to the momenta their quantum-mechanical operators (of course, with the appropriate clarification $\partial \rightarrow \hat{\partial}$ ), we obtain again that, in general, the derivatives do not commute $\left[\frac{\hat{\partial}}{\hat{\partial} x_{\mu}}, \frac{\hat{\partial}}{\partial x_{\nu}}\right] \neq 0$.

Furthermore, since the energy derivative corresponds to the operator of time and the $i$-component momentum derivative, to $\hat{x}_{i}$, we put forward the following Ansatz in the momentum representation:

$$
\left[\hat{x}^{\mu}, \hat{x}^{\nu}\right]=\omega(\mathbf{p}, E(\mathbf{p})) F_{\|}^{\mu \nu} \frac{\partial}{\partial E},
$$

with some weight function $\omega$ being different for different choices of the antisymmetric tensor spin basis. In the modern literature the relation $\left[x^{\mu}, x^{\nu}\right] \sim$ $F^{\mu \nu}$, the electromagnetic tensor, is frequently used [10]. However, the idea of the broken Lorentz invariance by this method [11] concurs with the idea of the fundamental length, first introduced by V. G. Kadyshevsky [12] on the basis of old papers by M. Markov. Both ideas and corresponding theories are extensively discussed, e.g. [13]. In my opinion, the main question is: what is the space scale, when the relativity theory becomes incorrect.

\section{Conclusions}

We found that the commutator of two derivatives may be not equal to zero. As a consequence, for instance, the question arises, if the derivative $\hat{\partial}^{2} f / \hat{\partial} p^{\nu} \hat{\partial} p^{\mu}$ is equal to the derivative $\hat{\partial}^{2} f / \hat{\partial} p^{\mu} \hat{\partial} p^{\nu}$ in all cases? ${ }^{2}$ The pre-

\footnotetext{
${ }^{2}$ The same question can be put forward when we have differentiation with respect to the coordinates too, that may have impact on the correct calculations of the problem of accelerated charge in classical electrodynamics.
} 
sented consideration permits us to provide some bases for non-commutative field theories and induces us to look for further development of the classical analysis in order to provide a rigorous mathematical basis for operations with functions which have both explicit and implicit dependencies.

I am grateful to participants of conferences where this idea has been discussed [14]. I greatly apreciate the referee clear reports. I am grateful to R. Keys for his help with the French translation.

\section{References}

[1] H. Snyder, Phys. Rev. 71, 38 (1947); ibid. 72, 68 (1947).

[2] S. Doplicher, K. Fredenhagen and J. E.Roberts, Phys. Lett. B331, 39 (1994); S. Majid and H. Ruegg, Phys. Lett. B334, 348 (1994); J. Lukierski, H. Ruegg and W. J. Zakrzewski, Ann. Phys. 243, 90 (1995); N. Seiberg and E. Witten, JHEP 9909, 032 (1999), hep-th/9908142; I. F. Riad and M. M. Sheikh-Jabbari, JHEP 08, 045 (2000), hep-th/0008132; M. M. Sheikh-Jabbari, Phys. Rev. Lett. 84, 5265 (2000), hep-th/0001167; J. Madore, S. Schraml, P. Schupp and J. Wess, Eur. Phys. J. C16, 161 (2000).

[3] S. I. Kruglov, Ann. Fond. Broglie 27, 343 (2002), hep-th/0110059; B. G. Sidharth, Ann. Fond. Broglie 27, 333 (2002), physics/0110040.

[4] F. Dyson, Am. J. Phys. 58, 209 (1990). S. Tanimura, Ann. Phys. 220, 229 (1992); M. Land, N. Shnerb and L. Horwitz, hep-th/9308003.

[5] K. R. Brownstein, Am. J. Phys. 67, 639 (1999).

[6] L. D. Landau and E. M. Lifshitz, The Classical Theory of Fields. 4th revised English ed. [Translated from the 6th Russian edition] (Pergamon Press, 1979). 402p., §63.

[7] L. Škovrlj and T. Ivezić, hep-ph/0203116.

[8] J. D. Jackson, hep-ph/0203076.

[9] M. Jacob and G. C. Wick, Ann. Phys. 7, 404 (1959); H. M. Ruck and W. Greiner, J. Phys. G: Nucl. Phys. 3, 657 (1977).

[10] A. Connes, M. R. Douglas, and A. Schwarz, JHEP 02, 003 (1998), hep-th/9711162; D. Galtsov and V. Dyadichev, hep-th/0012059.

[11] S. M. Carroll, J. A. Harvey, V. A. Kosteleck, C. D. Lane and T. Okamoto, Phys. Rev. Lett. 87, 141601 (2001). 
[12] V. G. Kadyshevsky, Nucl. Phys. B141, 477 (1978); V. G. Kadyshevsky, M. D. Mateev, R. M. Mir-Kasimov and I. P. Volobuev, Theor. Math. Phys. 40, 800 (1979) [Teor. Mat. Fiz. 40, 363 (1979)]; V. G. Kadyshevsky and M. D. Mateev, Phys. Lett. B106, 139 (1981); V. G. Kadyshevsky and M. D. Mateev, Nuovo Cim. A87, 324 (1985); A. D. Donkov, R. M. Ibadov, V. G. Kadyshevsky, M. D. Mateev and M. V. Chizhov, ibid. 87, 350 (1985); ibid. 87, 373 (1985). See also the old work: V. G. Kadyshevsky, R. M. Mir-Kasimov and N. B. Skachkov, Yad. Fiz. 9, 212 (1969). See also references to the old works of M. Markov in the above-cited papers.

[13] A. Kempf, G. Mangano and R. B. Mann, Phys. Rev. D52, 1108 (1995); G. AmelinoCamelia, Nature 408, 661 (2000); gr-qc/0012051; hep-th/0012238; gr-qc/0106004; J. Kowalski-Glikman, hep-th/0102098; G. Amelino-Camelia and M. Arzano, hepth/0105120; N. R. Bruno, G. Amelino-Camelia and J. Kowalski-Glikman, hepth/0107039.

[14] V. V. Dvoeglazov, AIP Conf. Proc. 1191, 80 (2009). 


\title{
NOTE ADDED: \\ Question on Iterated Limits in Relativity \\ V. V. Dvoeglazov \\ UAF, Universidad de Zacatecas \\ Apartado Postal 636, Suc. 3 \\ Zacatecas 98061 Zacatecas, México
}

\begin{abstract}
ABSTRACT. Two iterated limits are not equal each other, in general. Thus, we present an example when the massless limit of the function of $E, \mathbf{p}, m$ does not exist in some calculations within quantum field theory.

RESUMÉ. Deux limites itérées ne sont pas égales en général. Ainsi, nous présentons un exemple oú la limite sans masse de la fonction de $E, \mathbf{p}, m$ n'existe pas dans certains calculs de la théorie quantique des champs.

KEYWORDS: Iterated limits, Relativity, Massless limit.
\end{abstract}

In the previous paper [1] we found some intrinsic contradictions related to the mathematical foundations of modern physics. It is well known that the partial derivatives commute in the Minkowski space (as well as in the 4-dimensional momentum space). However, if we consider that the energy is the implicit function of the 3-momenta and mass (thus, approaching the mass hyperboloid formalism, $E^{2}-\mathbf{p}^{2} c^{2}=m^{2} c^{4}$ ) then we may be interested in the commutators of the whole-partial derivatives [2] instead. The whole-partial derivatives do not commute, in general. If they are associated with the corresponding physical operators, we would have the uncertainty relations for dynamically-conjugated physical quantities in the latter case. This is an intrinsic contradiction. While we start from the same postulates, on using two different ways of reasoning we arrive at the two different physical conclusions.

In the present note I would like to ask another question related to the mathematical foundations of the special relativity. Sometimes, when calculating dynamical invariants (and other physical quantities in quantum field theory), and when studying the corresponding massless limits we need to calculate the iterated limits. We may encounter a rare situation when two iterated limits are not equal each other in physics!? See, for example, Ref. [3]. We were puzzled calculating the iterated limits of the aggregate $\frac{E^{2}-\mathbf{p}^{2}}{m^{2}}$ (or the inverse one, $\left.\frac{m^{2}}{E^{2}-\mathbf{p}^{2}}, \quad c=1\right){ }^{3}$

$$
\lim _{m \rightarrow 0} \lim _{E \rightarrow \pm \sqrt{\mathbf{p}^{2}+m^{2}}}\left(\frac{m^{2}}{E^{2}-\mathbf{p}^{2}}\right)=1
$$

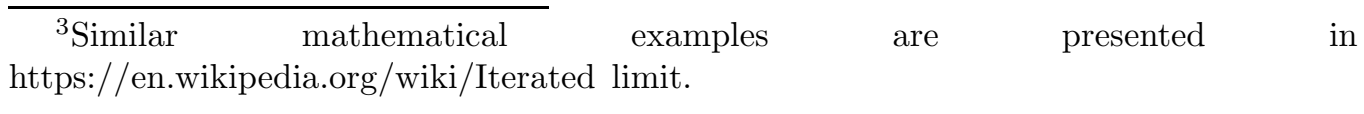




$$
\lim _{E \rightarrow \pm \sqrt{\mathbf{p}^{2}+m^{2}}} \lim _{m \rightarrow 0}\left(\frac{m^{2}}{E^{2}-\mathbf{p}^{2}}\right)=0 .
$$

Physics should have the well-defined dynamical invariants. Which iterated limit should be applied in the study of massless limits?

The question of the iterated limits is studied in $[4,5]$. However, the answers leave the room for misunderstandings and contradictions with the experiments.

I am grateful to the referee for his clear reports. I am grateful to R. Keys for his help with the French translation.

\section{References}

[1] V. V. Dvoeglazov, Phys. Essays, this issue.

[2] K. R. Brownstein, Am. J. Phys. 67, 639 (1999).

[3] V. V. Dvoeglazov. Int. J. Theor. Phys. 37, 1915 (1998); Czech. J. Phys. 50, 225 (2000).

[4] V. A. Ilyin, V. A. Sadovnichii and B. Kh. Sendov, Mathematical Analysis. Continuation. (MSU, Moscow, 1987), in Russian.

[5] V. A. Ilyin and E. G. Poznyak, Fundamentals of Mathematical Analysis. Vol. 1. Ch. 14. Functions of Several Variables (Fizmatlit, Moscow, 2002), in Russian. 